\title{
On the natural completion functor for Cauchy spaces
}

\section{R. Frič and D.C. Kent}

The natural completion functor on the category of $c^{\wedge}$-embedded Cauchy spaces induces a completion functor on a category of sequential Cauchy spaces which preserves sequential regularity.

\section{Introduction}

This work is a continuation of the Cauchy space completion theory developed in [3], and the reader is asked to refer to this source for terminology and notation used without being defined in this paper. The assumption is made throughout this paper that all Cauchy spaces are Cauchyseparated unless otherwise indicated.

In Section 2, we show that the natural completion developed in [3] determines a completion functor, denoted by $N$, on the category of $\mathrm{CCH}$ of $c^{\wedge}$-embedded Cauchy spaces which preserves such properties as total boundedness and uniformizability. In section 3 , it is shown that the category $\mathrm{SCH}$ of sequential Cauchy spaces is a subcategory of $\mathrm{CCH}$, and that $N$ is a completion functor when restricted to $\mathrm{SCH}$. In Section 4, the functor $N$ is composed with two modification functors to produce a completion functor $N_{S}$ on the category SRCH of sequentially regular Cauchy spaces.

\section{The completion functor $N$}

The term "convergence space" will be identified with "complete Cauchy space". Thus every convergence space $(X, q)$ is $\omega$-Hausdorff and

Received 19 January 1978. 
satisfies Fischer's axiom

$$
(F \rightarrow x \text { and } G \rightarrow x \text { implies } F \cap G \rightarrow x) \text {. }
$$

Let CHY be the category with Cauchy spaces as objects and Cauchycontinuous maps as morphisms. Let $\mathrm{LCH}$ be any full subcategory of CHY, and let $\mathrm{LCH}^{*}$ be the full subcategory of complete objects in $\mathrm{LCH}$. The Extension of Identity Principle states that two continuous mappings of a convergence space into a Hausdorff convergence space which agree on a dense subset are identical; from this it follows that a morphism $f:(X, C) \rightarrow(Y, D)$ in $\mathrm{CHY}$ is an epimorphism if $f(X)$ is dense in $(Y, D)$.

DEFINITION 2.1. A completion functor $T$ on $\mathrm{LCH}$ is a covariant functor $T: \mathrm{LCH} \rightarrow \mathrm{LCH}^{*}$ with the following properties:

(1) for each $(X, C) \in L C H$, there is an embedding $i_{T}:(X, \mathcal{C}) \rightarrow T(X, \mathcal{C})$ such that $i_{T}(X)$ is dense in $T(X, C)$;

(2) if $f$ is a Cauchy-continuous mapping of $(X, C)$ into $(Y, D) \rightarrow \mathrm{LCH}^{*}$, then there is a unique Cauchy-continuous mapping $\bar{f}$ of $T(X, C)$ into $(Y, D)$ such that the following diagram commutes;

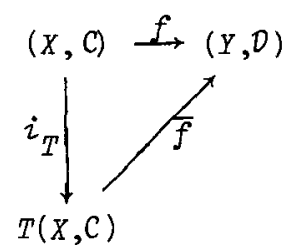

Then $\left(T(X, \mathcal{C}), i_{T}\right)$ is called a $T$-completion of $(X, C)$.

In other words, $i_{T}$ is an epi-reflection of $L C H$ into $\mathrm{LCH}^{*}$. Note that $T(X, C)$ is (up to a commuting homeomorphism) uniquely determined. If $g:\left(x_{1}, C_{1}\right) \rightarrow\left(x_{2}, C_{2}\right)$ is a morphism in LCH, then $T g=\bar{g}$, where $\bar{g}: T\left(X_{1}, C_{1}\right) \rightarrow T\left(X_{2}, C_{2}\right)$ is the unique Cauchy-continuous extension whose existence is guaranteed by (2). The same functor symbol is used to represent transformations of both objects and morphisms; we shall also use the same functor symbol when the domain of the functor is restricted to a 
subcategory .

Let $\mathrm{CCH}$ be the full subcategory of CHY consisting of the $c^{\wedge}$-embedded Cauchy spaces. If $(X, C)$ is an object in $\mathrm{CCH}$, let $N(X, \mathcal{C})=\operatorname{hom}^{\wedge} C^{\wedge}(X, C)$ be the natural completion described in [3]. If $f:\left(x_{1}, C_{1}\right) \rightarrow\left(x_{2}, C_{2}\right)$ is a morphism in $C C H$, define $N(f)=\bar{f}$, where $\bar{f}: N\left(x_{1}, c_{1}\right) \rightarrow N\left(X_{2}, c_{2}\right)$ is the unique Cauchy-continuous extension of $f$ whose existence is established by Theorem 2.4 of [3]. Using these and other results from [3], we immediately obtain the following result.

PROPOSITION 2.2. $N$ is a completion functor on $\mathrm{CCH}$.

The functor $N$ will be called the natural completion functor. For any $(X, C) \in \mathrm{CCH}, N(X, C)$ is known to be a c-embedded convergence space; in particular $N(X, C)$ is Hausdorff and $\omega$-regular. The latter property is stronger than ordinary regularity for convergence spaces. In the remainder of this section, we examine some additional properties of the functor $N$.

A Cauchy space is said to be totally bounded if each ultra-filter is a Cauchy filter.

LEMMA 2.3. A regular completion of a totally bounded Cauchy space is totally bounded.

Proof. Let $\left(\left(X^{*}, \mathcal{C}^{*}\right), j\right)$ be a regular completion of $(X, \mathcal{C})$. If $G$ is an ultrafilter on $X^{*}$, then $\operatorname{cl} j(X)=X^{*}$ implies that there is an ultrafilter $F$ on $X^{*}$ containing $j(X)$ such that $G \geq$ cl $F$. If $(X, C)$ is totally bounded, then $F^{\prime}$, the restriction of $F$ to $X$, is in $C$, and so $j\left(F^{\prime}\right)=F \in C^{*}$. Since $\left(X^{*}, C^{*}\right)$ is regular, cl $F \in C^{*}$, and therefore $G \in C^{*}$.

A Cauchy space $(X, C)$ is said to be uniformizable if $C$ is the set of all Cauchy filters for some uniformity on $X$, and completely regular if $(X, C)$ is a Cauchy subspace of a completely regular topological space (considered as a complete Cauchy space). If $\mathrm{UCH}$ and $\mathrm{QCH}$ are the full subcategories of CHY consisting of the uniformizable and completely regular Cauchy spaces, respectively, then it is clear that $\mathrm{UCH} \subset \mathrm{QCH} \subset \mathrm{CCH}$. Since there are completely regular topological spaces which have no compatible complete uniformity, a completely regular Cauchy space need not be uniformizable. 
An object in either $\mathrm{UCH}$ or $\mathrm{QCH}$ has a strict regular completion within its own category. If $(X, C) \in \mathrm{QCH}$ then such a completion is obtained by taking the closure of $(X, C)$ in the completely regular topological space in which $(X, C)$ is, by definition, embedded. For $(X, C) \in U C H$, a strict regular completion in $U C H$ can be similarly obtained by first completing the associated uniformity.

THEOREM 2.4. The completion functor $N$ preserves total boundedness, complete regularity, and uniformizability.

Proof. For total boundedness, the assertion follows from Lemma 2.3. For $(X, C) \in Q C H$, let $\left(X^{*}, C^{*}\right)$ denote the strict completion of $(X, C)$ described in the preceding paragraph. It follows from Proposition 1.5, [3], that $N(X, \mathcal{C})$ and $N\left(X^{*}, C^{*}\right)$ are homeomorphic spaces. Also, since $\left(X^{*}, C^{*}\right)$ is complete and $\hat{C}^{\wedge}$-embedded, $N\left(X^{*}, C^{*}\right)=\left(X^{*}, C^{*}\right)$. Therefore, $N(X, C)$ is completely regular. The same reasoning is applicable if $(X, C) \in \mathrm{UCH}$.

From Theorem 2.4, it follows that every Hausdorff compactification of a completely regular topological space can be obtained by choosing an appropriate Cauchy structure and applying the natural completion functor.

\section{Sequential Cauchy spaces}

One branch of convergence space theory is concerned with sequences rather than filters. The Cauchy space axioms can be readily adapted to the sequential setting, and indeed this has been done in [1]. Since we are interested in using the natural completion functor $N$ as a tool for studying completions of sequential Cauchy spaces, it is more convenient in this case to axiomatize sequential Cauchy spaces by means of filters generated by sequences. We shall refer to any filter generated by a sequence as a sequential filter.

DEFINITION 3.1. A Cauchy $(X, \mathcal{C})$ is sequential if, for each $F \in \mathcal{C}$, there is a sequential filter $G \in \mathcal{C}$ such that $G \leq F$.

A complete sequential Cauchy space can be taken as the definition of a sequential convergence space. Let $\mathrm{SCH}$ denote the full subcategory of $\mathrm{CHY}$ consisting of sequential Cauchy spaces.

PROPOSITION 3.2. Each sequential Cauchy space has a strict 
sequential completion.

Proof. Let $X^{*}$ be the set of all Cauchy equivalence classes of members of $C$, and define $j: X \rightarrow X^{*}$ by: $j(x)=[\dot{x}]$, the equivalence class containing the fixed ultrafilter generated by $\{x\}$. Define a convergence structure $q$ on $X^{*}$ as follows:

(1) if $[\dot{x}] \in j(X)$, then a filter $H$ q-converges to $[\dot{x}]$ if and only if there is a sequential filter $F \rightarrow x$ in $(X, C)$ such that $H \geq j(F)$;

(2) if $\alpha \in X^{*}-j(X)$, then $H \quad q$-converges to $\alpha$ if and only if there is a sequential filter $F \rightarrow \alpha$ such that $H \geq j(F) \cap \dot{\alpha}$.

One can easily verify that $\left(X^{*}, q\right)$ is a sequential convergence space in which $(X, C)$ is densely embedded by the mapping $j$. If $C^{*}$ is the complete Cauchy structure on $X^{*}$ consisting of the $q$-convergent filters, then $\left(\left(X^{*}, C^{*}\right), j\right)$ is thus a sequential completion of $(X, \mathcal{C})$. The completion is trivially strict, since the only members of $C^{*}$ containing $X^{*}-j(X)$ are fixed ultrafilters.

COROLLARY 3.3. The sequential Cauchy spaces are precisely the Cauchy subspaces of the sequential convergence spaces.

THEOREM 3.4. $\mathrm{SCH} \subset \mathrm{CCH}$.

Proof. If $(X, C) \in \mathrm{SCH}$, then it is shown in the preceding proof that $(X, C)$ is a Cauchy subspace of a sequential convergence space. What we are calling a "sequential convergence space" is described in [2] as an " $\omega$-Hausdorff S-space satisfying Fischer's axiom", and it is shown in [2] that such spaces are c-embedded convergence spaces. From [3], it follows that a c-embedded convergence space is a complete $c^{\wedge}$-embedded Cauchy space, and hence a member of $\mathrm{CCH}$. Since the $c^{\wedge}$-embedded property is hereditary, $(X, C) \in \mathrm{CCH}$.

Next, we consider the behavior of the completion functor $N$ on the category $\mathrm{SCH}$. It is not known whether the completion $N(X, \mathcal{C})$ is strict for an arbitrary object $(X, \mathcal{C}) \in \mathrm{CCH}$, but it turns out that this completion is strict if $(X, C) \in S C H$. This fact is established with the help of the following lemma, which shows that even in the general case the functor $N$ exhibits a weak form of "strictness". 
LEMMA 3.5. Let $(X, C) \in \mathrm{CCH}$, Let $N(X, C)=\left(X_{N}, C_{N}\right)$, and let $i_{N}:(X, C) \rightarrow\left(X_{N}, C_{N}\right)$ be the natural embedding. Also, let $q$ be the convergence structure on $X_{N}$ determined by $C_{N}$. Then, if $F \rightarrow y$ in $\left(X_{N}, C_{N}\right)$, there is a fizter $G \rightarrow y$ in $\left(X_{N}, C_{N}\right)$ such that $i_{N}(X) \in G$, and $F \geq \mathrm{cl}_{\omega q} G$.

Proof. Define a convergence structure $p$ on $X_{N}$ as follows: $F \rightarrow y$ in $\left(X_{N}, p\right)$ if there is a filter $G \rightarrow y$ in $\left(X_{N}, c_{N}\right)$ such that $i_{N}(X) \in G$, and $F \geq c_{\omega q} G$. Clearly $q \leq p$, and it is easy to verify that $\omega p=\omega q$. Thus, if $D$ is the complete Cauchy structure on $X_{N}$ consisting of all $p$-convergent filters, it follows $(X, D)$ is a $e^{\wedge}$ embedded completion of $(X, C)$. Thus $i_{N}:(X, C) \rightarrow\left(X_{N}, D\right)$ has a Cauchy-continuous extension $\bar{i}_{N}:\left(X_{N}, C_{N}\right) \rightarrow\left(X_{N}, 0\right)$; this implies $C_{N} \subset D$, which is equivalent to $p \leq q$. Therefore $D=C_{N}$, and it follows that $N(X, C)$ satisfies the assertion of the lemma.

THEOREM 3.6. If $(X, C) \in \mathrm{SCH}$, then $N(X, C) \in \mathrm{SCH}$. Indeed, $N(X, C)$ is a strict completion which is.homeomorphic to the completion $\left(X^{*}, C^{*}\right)$ described in Proposition 3.2.

Proof. The first assertion follows from the second. To prove the second, it is sufficient to show that the completion $N(X, C)$ is strict, since the completion of Proposition 3.2 is also strict and regular, and it follows from Corollary 1.6 of [4] that two strict regular completions of the same Cauchy space are homeomorphic.

To verify that $N(X, \mathcal{C})$ is strict, we refer to the notation of Lemma 3.5. Let $F \rightarrow y$ in $\left(X_{N}, C_{N}\right)$. If $(X, \mathcal{C}) \in \mathrm{SCH}$, then we may assume that the filter $G$ described in the lemma is sequential. Then it is easy to see that ${ }^{\mathrm{cl}}{ }_{\omega q} G=\mathrm{cl}_{q} G=G \cap \dot{y}$. Since $G \rightarrow y$ in $\left(x_{N}, c_{N}\right)$ and $F \geq \mathrm{cl}_{q} G$, it follows that $N(X, \mathcal{C})$ is a strict completion of $(X, C)$.

As a supplement to Theorem 2.4, we note that the completion functor $N$ also preserves the sequential property. 


\section{Sequentially regular Cauchy spaces}

A sequential convergence space $(X, q)$ is said to be sequentially regular if $(X, q)$ has the same convergence relative to sequential filters as does $(X, \omega q)$, where $\omega q$ is the completely regular modification of $q$. Sequential regularity is important in the construction of Novak's sequential envelope (see [5] and [6]). We extend this concept to sequential Cauchy spaces, and use a "translation" of the functor $N$ to obtain a completion functor $N_{S}$ on the category of sequentially regular Cauchy spaces which, under certain conditions, yields Novák's sequential envelope as its completion (see Example 4.6).

In defining sequential regularity for Cauchy spaces, we make use of the two modification functors defined below.

DEFINITION 4.7. The sequential modification functor $S$ is a covariant functor $S: \mathrm{CCH} \rightarrow \mathrm{SCH}$ defined as follows: for an object $(X, C) \in \mathrm{CCH}, S(X, \mathcal{C})=\left(X, C_{S}\right)$, where $G \in \mathcal{C}_{S}$ means that $G \in \mathcal{C}$ and there is a sequential filter $F \in \mathcal{C}$ such that $F \leq G$; for a morphism $f \in \mathrm{CCH}, S f=f$.

In other words, $S(X, C)$ has the coarsest sequential Cauchy structure on the same underlying set which is finer than $\mathcal{C}$.

DEFINITION 4.2. The completely regular modification functor $Q$ is a covariant functor $Q: \mathrm{CCH} \rightarrow \mathrm{QCH}$ defined as follows: for an object $(X, C) \in C C H, Q(X, C)=\left(X, C_{Q}\right)$, where $C_{Q}$ is the finest completely regular Cauchy structure on $X$ coarser than $C$; for a morphism $f \in \mathrm{CCH}, Q f=f$.

The space $Q(X, C)$ can be described as follows: let $N(X, \mathrm{C})=\left(X_{N}, \mathcal{C}_{N}\right)$, and let $q$ be the convergence structure corresponding to the complete Cauchy structure $\mathcal{C}_{N}$; if we assume that $(X, C)$ is a subspace of $\left(X_{N}, q\right)$, then $Q(X, C)$ is the restriction of $\left(X_{N}, w q\right)$ to the set $X$. It follows that $Q(X, C)$ is a dense Cauchy subspace of the completely regular topological space $\left(X_{N}, \omega q\right)$, which can also be described as $Q N(X, \mathcal{C})$, and consequently $N Q(X, C)=Q N(X, C)$. Thus we have established the following result. 
PROPOSITION 4.3. For any object $(X, C) \in \mathrm{CCH}$, $Q N(X, C)=N Q(X, C)$.

DEFINITION 4.4. A sequential Cauchy space $(X, C)$ is sequentially regular if $S Q(X, \mathcal{C})=(X, \mathcal{C})$.

Let SRCH denote the full subcategory of $\mathrm{SCH}$ whose objects are the sequentially regular Cauchy spaces. Note that $S Q(X, C) \in S R C H$ for any object $(X, C) \in \mathrm{SCH}$; thus the composite functor $S Q$ may be regarded as the "sequentially regular modification functor" which maps SCH into $\mathrm{SRCH}$.

Next we define the functor $N_{S}: \mathrm{SRCH} \rightarrow \mathrm{SRCH}$ as follows: $N_{S}$ is the restriction of the composite functor SNQ to the category SRCH - Note that $N_{S}(X, C)=S N Q(X, C)=S Q N(X, C)$ by Proposition 4.3; therefore $N_{S}(X, \mathcal{C}) \in \mathrm{SRCH}$, since $N(X, \mathcal{C}) \in \mathrm{SCH}$ by Theorem 3.5 .

THEOREM 4.5. $N_{S}$ is a completion functor on SRCH.

Proof. Let $(X, C) \in S R C H$. As noted in the discussion following Definition 4.2, $Q(X, C)$ is a dense subspace of $Q N(X, C)$, and one can routinely verify that $(X, C)=S Q(X, C)$ is a dense subspace of $S Q N(X, C)=N_{S}(X, C)$. Both of the functors $S$ and $Q$ preserve completeness, and so $N_{S}(X, \mathrm{C}) \in \mathrm{SRCH} *$ and $\left(N_{S}(X, \mathcal{C}), i_{N}\right)$ is a completion of $(X, C)$. To verify the universal property, let $f:(X, C) \rightarrow\left(X_{1}, C_{1}\right)$ be Cauchy-continuous, with $(X, C) \in \operatorname{SRCH}$ and $\left(X_{1}, C_{1}\right) \in \mathrm{SRCH}^{*}$. In the following commutative diagram, $\bar{f}=N f$, all maps are morphisms, and each unlabelled map is the identity function:

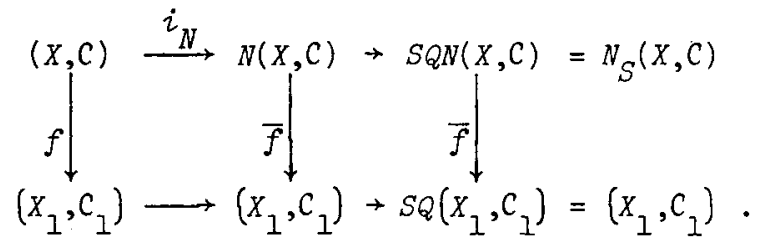

This completes the proof.

EXAMPLE 4.6. Let $(X, q)$ be a sequentially regular convergence space. A filter $F$ on $X$ is said to be fundamental if $f(F)$ is 
convergent for each real-valued continuous function $f$ on $(X, q)$. Let $C$ be the Cauchy structure on $X$ consisting of all filters $G$ on $X$ which are finer than some fundamental sequential filter $F$; it follows that $(X, \mathcal{C}) \in \mathrm{SRCH}$. In this case, $Q(X, C)=\left(X, C_{Q}\right)$ has as its Cauchy filters the set of all fundamental filters on $X$. It can be shown that $N_{S}(X, \mathcal{C})$ is a subspace of Novák's sequential envelope $\sigma(X, \mathcal{C})$, and coincides with $\sigma(X, C)$ whenever $(X, \mathcal{C})$ is dense (in our sense) in $\sigma(X, C)$.

It should be noted that the completion functor $N_{S}$ on SRCH is not strict. Indeed, no strict completion functor exists on SRCH. The natural completion is, up to homeomorphism, the only strict completion of a sequential Cauchy space, and it does not preserve sequential regularity.

\section{References}

[1] R. Frič and D.C. Kent, "Completion of sequential Cauchy spaces", Corment. Math. Univ. Carolinae 18 (1977), 351-361.

[2] R. Frič and D.C. Kent, "On c-embedded sequential convergence spaces", submitted.

[3] R.J. Gazik and D.C. Kent, "Regular completions of Cauchy spaces via function algebras", Bul2. Austral. Math. Soc. 11 (1974), 77-88.

[4] D.C. Kent and G.D. Richardson, "Regular completions of Cauchy spaces", Pacific J. Math. 51 (1974), 483-490.

[5] Václav Koutník, "On sequentially regular convergence spaces", Czechoslovak Math. J. 17 (92) (1967), 232-247.

[6] Josef Novâk, "On convergence spaces and their sequential envelopes", Caechoslovak Math. J. 15 (90) (1965), 74-100.

Vysoká škola dopravná Katedra matematiky F SET, Žilina, Zeskoslovensko;
Department of Pure and Applied Mathematics, Washington State University, Pul Iman, Washington, USA. 